\title{
Molecular Characterization of a Dual Endothelin-1/Angiotensin II Receptor
}

\author{
Nelson Ruiz-Opazo, ${ }^{1}$ Kenji Hirayama, ${ }^{1}$ Kaoru Akimoto, ${ }^{1}$ and \\ Victoria L. M. Herrera ${ }^{1,2}$ \\ ${ }^{1}$ Section of Molecular Genetics, Whitaker Cardiovascular Institute, \\ and ${ }^{2}$ Section of Cardiology, Department of Medicine, Boston \\ University School of Medicine, Boston, Massachusetts, U.S.A. \\ Communicated by S. H. Orkin. Accepted December 16, 1997.
}

\begin{abstract}
Background: The molecular recognition theory (MRT) provides a conceptual framework that could explain the evolution of intermolecular and intramolecular interaction of peptides and proteins. As such, it predicts that binding sites of peptide hormones, and its receptor binding sites were originally encoded by and evolved from complementary strands of genomic DNA.

Materials and Methods: On the basis of principles underlying the MRT, we screened a rat brain complementary DNA library using an AngII followed by an endothelin-1 (ET-1) antisense oligonucleotide probe, expecting to isolate potential cognate receptors.

Results: An identical cDNA clone was isolated independently from both the AngII and ET-1 oligonucleotide screenings. Structural analysis revealed a receptor polypeptide containing a single predicted transmembrane region with distinct ET-1 and AngII putative binding domains. Functional analysis demonstrated ET-1-
\end{abstract}

and AngII-specific binding as well as ET-1- and AngIIinduced coupling to a $\mathrm{Ca}^{2+}$ mobilizing transduction system. Amino acid substitutions within the predicted ET-1 binding domain obliterate ET-1 binding while preserving AngII binding, thus defining the structural determinants of ET-1 binding within the dual ET-1/AngII receptor, as well as corroborating the dual nature of the receptor. Conclusions: Elucidation of the dual ET-1/AngII receptor provides further molecular genetic evidence in support of the molecular recognition theory and identifies for the first time a molecular link between the ET-1 and AngII hormonal systems that could underlie observed similar physiological responses elicited by ET-1 and AngII in different organ systems. The prominent expression of the ET-1/AngII receptor mRNA in brain and heart tissues suggests an important role in cardiovascular function in normal and pathophysiological states.

\section{Introduction}

The hydropathic character of an amino acid appears to be determined by the second base of a particular codon (1). A second base, U, generally specifies hydrophobic amino acids, while a second base, A, specifies hydrophilic amino acids (1). Since A and U are complementary, amino acid sequences derived from complementary

Address correspondence and reprint requests to: Dr. Nelson Ruiz-Opazo, Section of Molecular Genetics, W-609, Whitaker Cardiovascular Institute, Boston University School of Medicine, 700 Albany Street, Boston, MA 02118, U.S.A. Phone: (617) 638-4384; Fax: (617) 638-4066.
DNA strands will generate peptides of inverted patterns of hydropathy. This fundamental observation provides the basis for the molecular recognition theory, which hypothesizes that complementary nucleotide sequences specify peptides that could interact through complementary structures as a result of their exactly inverted pattern of amino acid hydropathy (1). This theory further suggests that binding sites of interacting proteins, such as peptide hormone and receptors (2), and peptide substrate and enzyme (3), evolved from complementary strands of genomic DNA. This molecular paradigm fur- 
ther implies that the antisense peptide encoded by the antisense strand of peptide hormone mRNAs (spanning the hormone) represent the putative evolutionary precursor binding domain of corresponding peptide hormones. As a corollary, it can be envisioned that mutations in these precursor binding domains favoring an increased ligand-receptor affinity have occurred over time, leading to today's respective binding domains.

Numerous biochemical studies have reported experimental evidence in support of this theory $(4,5)$. However, the molecular recognition theory still remains controversial because of some negative biochemical and theoretical analysis $(6-10)$. Our recent report identifying the AngII/AVP receptor represents the first molecular genetic evidence in support of this theory (11). Our current studies were designed to further test this theory by screening a rat brain complementary DNA library using an AngII followed by an endothelin-1 (ET-1) antisense oligonucleotide probe to potentially isolate additional AngII and ET-1 receptor isoforms. As with the AngII/AVP receptor (11), an identical cDNA clone was isolated independently from both the AngII and ET-1 oligonucleotide screenings. Functional and mutational studies of the predicted polypeptide elucidates a novel dual ET-1/ AngII receptor, providing further molecular genetic evidence in support of the molecular recognition theory, as well as demonstrating for the first time a molecular link between the ET-1 and AngII hormonal systems. The identification of the structural determinants of hormone binding within the dual ET-1/AngII receptor provides new insight into the evolutionary origin of hormone-binding domains present in different isoreceptors.

\section{Materials and Methods}

\section{cDNA Screening and Nucleotide Sequencing}

An adult rat brain $\lambda$ gt $11 \mathrm{cDNA}$ library (Clontech) was screened twice independently with the ET-1 [5' - CCAGATGATGTCCAGGTGGCAGAAGTAGA CACACTCTTTATCCATCAGGGAAGAGCAGGAG CA-3'] (12) and AngII [5'-AAAGGGGTGGATGT ATACGCGGTC-3'] (13) antisense oligonucleotide probes. Hybridizations were done as described (11). After the first round of hybridization, a significant number of potential positive signals were detected. Of these, we proceeded to isolate five cDNA clones hybridizing to the AngII probe and three cDNA clones hybridizing to the
ET-1 probe. These clones showed the greatest signal to background ratio and they represented less than $5 \%$ of the potential positive signals. Among these clones we detected the $3.3-\mathrm{kb}$ cDNA isolated in both AngII and ET-1 screenings. The $3.3-\mathrm{kb}$ cDNAs were subcloned into M13 vectors and subsequently sequenced on both strands as described elsewhere (11). DNA sequence analysis was done using the GENEPRO computer DNA analysis program (Riverside Scientific Enterprises).

\section{Expression Studies in CosIpMAM-ET-1/AngII Transfectants}

The 756-bp SspI-EcoRI fragment spanning nucleotides 2518-3274 of the full-length ET-1/AngII receptor cDNA was subcloned directionally $\left(5^{\prime}-3^{\prime}\right)$ into the NheI site of the pMAMneo expression vector (Clontech). The pMAM-ET-1/ AngII expression vector was then transfected into Cosl cells and stable transfectants were selected and maintained in G418 for subsequent studies. Expression of mutant receptors was done similarly using the pMAM-ET-1/AngII R64G and pMAM-ET-1/AngII W65G expression vectors. ${ }^{45} \mathrm{Ca}^{2+}$ efflux assays were done essentially as previously described (14). Cell cultures (P 35 dishes) were equilibrated with ${ }^{45} \mathrm{Ca}^{2+}$ under standard growth conditions (DMEM $+10 \%$ $\mathrm{FCS}+250 \mu \mathrm{g} / \mathrm{ml} \mathrm{G} 418+5 \mu \mathrm{Ci} / \mathrm{ml}$ of ${ }^{45} \mathrm{CaCl}_{2}$ ) for 18 to $20 \mathrm{hr}$. Assays were initiated by removal of the ${ }^{45} \mathrm{Ca}^{2+}$ media and followed by rapid sequential washing and aspirations with three 1-ml aliquots of physiological buffer ( $\mathrm{PB}$ composition in mmol/L: $\mathrm{NaCl} 140 ; \mathrm{KCl} 5.4 ; \mathrm{CaCl}_{2}$ 1.8; $\mathrm{MgCl}_{2}$ 1.6; D-glucose 5.5; Hepes 5, $\mathrm{pH}$ 7.4). One milliliter of assay solution containing appropriate ligands dissolved in PB were promptly added and efflux was allowed to proceed for the specific time intervals. Efflux was terminated by rapid washing and aspiration with three $1-\mathrm{ml}$ aliquots of $\mathrm{MgCl}_{2}$-free $\mathrm{PB}$ containing $5 \mathrm{mmol} / \mathrm{L} \mathrm{LaCl}$. Residual ${ }^{45} \mathrm{Ca}^{2+}$ content was expressed relative to total isotope present in cultures that received the wash protocol without any intervening efflux interval (time $0=12,050 \pm 941 \mathrm{cpm}$ ). All assays were done at $37^{\circ} \mathrm{C} .{ }^{125} \mathrm{I}$-ET-1 binding experiments were performed on intact cells in Dulbecco's modified Eagle medium (DMEM) supplemented with $20 \mathrm{mmol} / \mathrm{L}$ Hepes, $\mathrm{pH} 7.3$ and $0.1 \%$ bovine serum albumin (BSA). Incubations were performed at $37^{\circ} \mathrm{C}$ for $60 \mathrm{~min}$. ${ }^{125} \mathrm{I}$-AngII binding experiments were performed as described elsewhere (11). Specific binding was determined as 
the difference between the total radioactivity bound to cells and the radioactivity bound to blanks containing $1 \mu \mathrm{mol} / \mathrm{L}$ ET- 1 or $1 \mu \mathrm{mol} / \mathrm{L}$ AngII. Affinity constants were determined by Scatchard analysis (RADLIG, Version 4 Program, McPherson).

\section{Immunocytochemistry}

A polyclonal rabbit antipeptide antibody (custom-made by Multiple Peptide Systems, San Diego, CA) was raised against the synthetic peptide $\mathrm{P}_{51}$ LLTSLGSKE $_{60}$ spanning a portion of the predicted extracellular domain of the ET-1/AngII receptor. Control CoslpMAMneo and test Cos 1pMAM-ET-1/AngII cells were reacted with this antibody (1:500) and immunostained as described previously (11).

\section{Site-Directed Mutagenesis}

Mutants were constructed by oligonucleotidedirected mutagenesis using the Transformer SiteDirected Mutagenesis Kit (Clontech) and the pMAM-ET-1/AngII756 as template. Mutagenic oligonucleotides were as follows: 5'-CCA-GTTCCA-GCC-AGA-CTT-CAT-CTC-3' (antisense strand) replacing the codon CGC $\left(\mathrm{R}_{64}\right)$ by GGC $\left(\mathrm{G}_{64}\right)$ and 5'-CCA-GTT-CCC-GCG-AGA-CTTCAT-CTC-3' (antisense strand) replacing the codon TGG $\left(W_{65}\right)$ by GGG $\left(G_{65}\right)$. Both mutants were verified by nucleotide sequencing of the entire amino acid coding region of the ET-1/ AngII receptor to ensure the absence of unwanted mutations. Mutant recombinants were called pMAM-ET-1/AngII R64G and pMAM-ET1/AngII W65G.

\section{Results}

Isolation of the Identical cDNA Clone from Independent AngII and ET-1 Screenings

The isolation of the ET-1/AngII receptor cDNA was accomplished by using the same strategy employed for the identification of the dual AngII/AVP receptor (11). In this particular case, we screened $0.5 \times 10^{6}$ recombinants from an adult rat brain cDNA library using a 24-base AngII antisense oligonucleotide probe followed by a 63-base ET-1 antisense oligonucleotide probe (see Materials and Methods). Unexpectedly, an identical cDNA clone, approximately $3.3 \mathrm{~kb}$ in length, was isolated independently from the AngII and ET-1 oligonucleotide screening. With the precedence of a dual AngII/AVP receptor (11), the logical prediction was that this $3.3-\mathrm{kb}$ brain cDNA might encode a dual ET-l/AngII receptor.

\section{Structural Analysis of the ET-1/AngII Receptor CDNA}

Nucleotide sequence analysis of the 3.3-kb ET-1/ AngII receptor cDNA revealed a single open reading frame (ORF) encoding a protein of 127 amino acids with a predicted molecular mass of 13,698 daltons (Fig. 1A). A single region with significant homology was found for each antisense peptide sequence in the 127 amino acid (aa) ORF. Comparison of the ET-1 and AngII cRNA (complementary RNA) sequences with the nucleotide and amino acid sequences of the ET-1/ AngII receptor identified $\mathrm{E}_{60} \mathrm{MKSRWNW}_{67}$ as a potential ET-1 binding domain (Fig. 1C), and $\mathrm{G}_{41} \mathrm{AASMQV}_{47}$ as a potential AngII binding domain (Fig. 1C) within the ET-1/AngII receptor. This analysis also delineates the antisense peptide from frame 2 as the putative evolutionary ET-1 precursor binding domain (Fig. 1C), whereas the antisense peptide from frame 3 is the putative evolutionary AngII precursor binding domain (Fig. 1C). Consistently, the single region with the highest nucleotide sequence homology to the AngII oligonucleotide probe $(63 \%$ identity spanning nucleotides [nt] 2802-2820) and the single region with the highest nucleotide sequence homology to the ET-1 oligonucleotide probe (65\% identity spanning nt 2859-2881) are distinct and correspond to the AngII and ET- 1 antisense peptide homology regions (Fig. 1). According to the molecular recognition theory, these antisense homology regions should identify putative AngII and ET-1 binding domains, respectively.

Hydropathy analysis using the KyteDoolittle scale (15) and GES hydropathy plot (16) predicts a single transmembrane domain (H-1; Fig. 1A). H-l ( $n=20$ amino acids) is predicted to cross the plasma membrane considering that a transmembrane region of 12-14 amino acids have been experimentally proven to be sufficient to cross the plasma membrane (17). The existence of a single transmembrane-spanning region in conjunction with the identification of the putative AngII and ET-1 binding domains within the amino terminal end predict the localization of the ET-1/AngII receptor amino terminal end to the extracellular side (Fig. 1B). Two potential phosphorylation sites for cAMP- 
TTTCTAAATGATTACTTTTCTAGATACCTGTTTACAAAACAGAAGATCCTCCCTT TGAAACCAAACTAAACTACACTTGAAGAATATAAAGT GCACAAAGGAAGACCACGATGAATCAGTACCACCCATC TTCCCAGCATTCAAGAATGTTCAGCGCACAGGAGGTGCACAGTAAGTGTTCCTGAGAGGAGTGGATACAACACTC AATTATCTGGGCATGTAATGCTGATCTGCGGTTTCCTTTACATCAGCCGGCCTCCTTCCCGGTTGGGATCAAGGA AGTGAACAGATGCAGACTCACTCTGGCAGGCACCACTGAGCCAGCCATTTACTCTCACTGCATAGCAAAGTTATT TTGTCAACTTGTTTCCAGGATCCTCTGCTTCCACAGAGCAGAAACACCTCGTCTAGGGGATTCTGATCCTTACCC TCTTCTTTACATTTCTCTCTCCAGAGAAGGTTATCCTCAGCCAAAATCCTCCAGTATCGACACGTCTGAGCCGCT TGCAGCAGGTCTTTGGGTTCCAGGAATGAAAGTACATAGAGTGCCAGCTATGGAAAAGGAAATAAGGGAGGCACA CTCAGACACATCACAGAAGAAAGGTTACTCTACGAAGGGTGAGCATTGAGCTGGGACTGCTGGATTTCAGTGGGC TGAACGAATCAAGAGAAGCAGCATTTTAAGAGCAAAGAAAATGCTCATCATTCTCACATTAGGAATCTGAGGCTT TACTCTGGGTAACCTGTGATTCTATTGGTATTTCCTTACAAAGTGAGAACAATGCCACTTATCACAAGTTTCTTG TGTGAGCCCAGTGCTCAAGCTCTTAGATAATCCAAATAAATGTTGATAAAGAGACTTTATATTGTTCATACCAAT TATCAAAAAATACAAGTACATTTCATGTCAGTGTGGTAATAATGTTTTAAATAACACACTTCCCTACAGGGTTAA GTCTATGCCATTATTCTTCCACGCAGACATAAAGCACTTCCCAAATGAAGAACACCCCAGTAGTCAGAAACAAAA ACCATAGCTGATATGCTAAGACAGGGCTCTCTCCTTTGTAACTTCTTTTTTCCTCAGGAAGTCTTTGAAAGAACA CAGAAGCCAAGAGAATCTTTTGGGGTTTTACCTTTTATTAATCATCTGTGCTTACTTCTTAAAATTCTAAAACAC TTTCAAATTTGGGGGACTGGTGATGGCTCAGTCAGTAAGGTTTCATGAAGATGAGGGCTCGGATCCTGGCAGTCT TGGAAAGTCAGGCATGGCAGTTCTAGCTATAGTCACTGCTCACTGGACAGCCAGCCACCGTAGCTAAAGGCGTAA GCTCCAGACTCAGTGAAAGACGACATGGCAAAAACAACATGGATCAGCTGAGGGATACACCTCTGGCCTCCACGG GCACATACGTGCAGGAGCATCTGAATGTACTTATGTATACCCACACGAATACATACACATCCTACACACATACAC ACTCTACAGGAGGTATCGGGCATGTAAGATAATCCAGACGAATATTCACTTCACGCCCTGATGGCAGCAAAGGGA TCTCGTGTTACTTTCATAAGTTTAGTCAAAGAGTTCTGATGTAGAAAAAGCTCACAAGAGCAAACACTTTTCTTC TGGGACACTGTCACCTTTAAAAAGTACTCAAAAGGGGGGAAAGTGCCAGGAAAAAGATGATTTATCAATTTGCTT TCCCCCAGAATTATATTTTAATTCATCAATTTTACTCAAATCTAATGCCAGATTCTAACTAGGACTATATTTAAT GCCACTAGGACTTTAGAGTGATCATCTAAGAAAGGAGAAAGCAAGACTCTTCCTGTTCAAATGAGGTTTCGGGAT CATCTGTATGAAGGATGTGGTAGTTTTTTGATGCTGTCTTTTTAACTGCTATTTATAACATGTGTATAGTAATTT GAGAAAATATGGACTATGGGGCATTATCTAATATCACATTATTTCTTCCTTTTGATAAAAATTAAGCTATGAAGT CTAATGTCAATATGTGCATTATATTTAAACCATCAGCCACACATGGCTGTATGACTAAGTGCCTAAGAATCCAAT TTTTTTGTGGTATCTCTCTCTCTCCCTCTCTCCGTATGTGTGTGTCTTTCTCTGTCTCTGTCTCTCTCTCTCTTT CTCTCTGACGAAGGATGATAAGTAGAAATGCCATAAAAACATATAGATAAATTTTATATATTGGGGGCTGGAGAG ATGGCTCAGTGGTTGAGAACACTGACTGTTCTTCAGAGGTCCTGAGTTAAATTCCCAGCAACCACATGGTAGCTC ACAACCATCTATATTGCATCTGATGCCCTCTTCTGGTGTGTCTAAAGACAGCTACCGGTATACTTACATATAATA AATAAATCTTTAAAAAAATTTTTATATATTAAAAAAAAATCACATAATGTAATAACCAGGAGAAATACGAACAA TCGATAAAATTACTGGTCTTGAAGGGGCATTAAATAATTAGCAAAATAAAAACAAAATTAATATTGTTGCTTAGT GAATCCAGAATTTTGAAAACATCCACTATATATAATAAACATACCAACTAACTAAAGTCAGCCTTTAGATAACCC AGGGAAAACT GAAGAGACTCGGCGACTTCACATGAAGCCTTACTTTATCCAAGCGGAAGAAAGCAGCACCTTGGT

ATGAGCACACTTTATGTAACAGCTGTACCAAAAAGCCACAGCAGTTTGCCAAAGTGTCAAGCCATGATGAGCAGG $\begin{array}{lllllllllllllllllllllllll}M & S & T & L & Y & V & T & A & V & P & K & S & H & S & S & L & P & K & C & Q & A & M & M & S & R\end{array}$

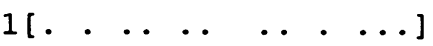

ACACTGCTTACAgGCATGGCTATGTATCTGGACAGCAGCCATGCGGGTGCTGCATCCATGCAGGTGAGCTGGCCG $\begin{array}{lllllllllllllllllllllllll}T & L & L & T & G & M & A & M & Y & L & D & S & S & H & A & G & A & A & S & M & Q & V & S & W & P\end{array}$ $2[\ldots \ldots+\ldots . . . . \quad . \quad$.

CCCTTACTCACCTCTTTGGGGAGCAAGGAGATGAAGTCTCGCTGGAACTGGGGCTCGATCACTTGCATCATGTGC

\begin{tabular}{llllllllllllllllllllllllll}
$P$ & $L$ & $L$ & $T$ & $S$ & $L$ & $G$ & $S$ & $K$ & $\mathbf{E}$ & $\mathbf{M}$ & $\mathrm{K}$ & $\mathbf{S}$ & $\mathbf{R}$ & $\mathbf{W}$ & $\mathbf{N}$ & $\mathbf{W}$ & $\mathrm{G}$ & $\mathbf{S}$ & $\mathrm{I}$ & $\mathrm{T}$ & $\mathrm{C}$ & $\mathrm{I}$ & $\mathbf{M}$ & $\mathrm{C}$ \\
\hline
\end{tabular}

TTCACTTGTGTGGGTTCACAGCTATCGATGAGCTCATCTAAGGCCAGCAACTTCTCTGGTCCACTCCAGCTCTAC $\begin{array}{llllllllllllllllllllllllllll}\mathbf{E} & \mathrm{T} & \mathrm{C} & \mathrm{V} & \mathrm{G} & \mathrm{S} & \mathrm{Q} & \mathrm{L} & \mathrm{S} & \mathrm{M} & \mathrm{S} & \mathrm{S} & \mathrm{S} & \mathrm{K} & \mathrm{A} & \mathbf{S} & \mathrm{N} & \mathrm{F} & \mathrm{S} & \mathrm{G} & \mathrm{P} & \mathrm{L} & \mathrm{Q} & \mathrm{L} & \mathrm{Y}\end{array}$

CAAAGAGGAATTGGACACATTACAAATCCATACAGAAGACCACCAGCACCTGCATGGCCATGTTCGAGCAGTGGA $\begin{array}{llllllllllllllllllllllllll}Q & R & G & I & G & H & I & T & N & P & Y & R & \mathbf{R} & \mathbf{P} & \mathbf{P} & \text { A } & \text { P } & \text { A } & \text { W } & \text { P } & \text { C } & \text { S } & \text { S } & \text { S } & \text { G } & 125\end{array}$

ACTACATGAAAGGGGACCGTGGACAGAGACCTTGTCTCCAGAAGCCACCAGAGCGATAGCAGTTTTTAGTTTCAG 3130 $\mathrm{T} T \star \mathrm{T}$

CAAGTTTACTCAGTACCTTTCCCGCAAAGCATTAAAAGTCATGACTGGCAGAAAAATAAGTCTGCATTTATTTTT AATTATAAGACTTATGCTAACACCAAGACACTGGGAGACACACAATATCCATCTGGGTTATTGACTAGG

Fig. 1. Continued on following two pages. 
B

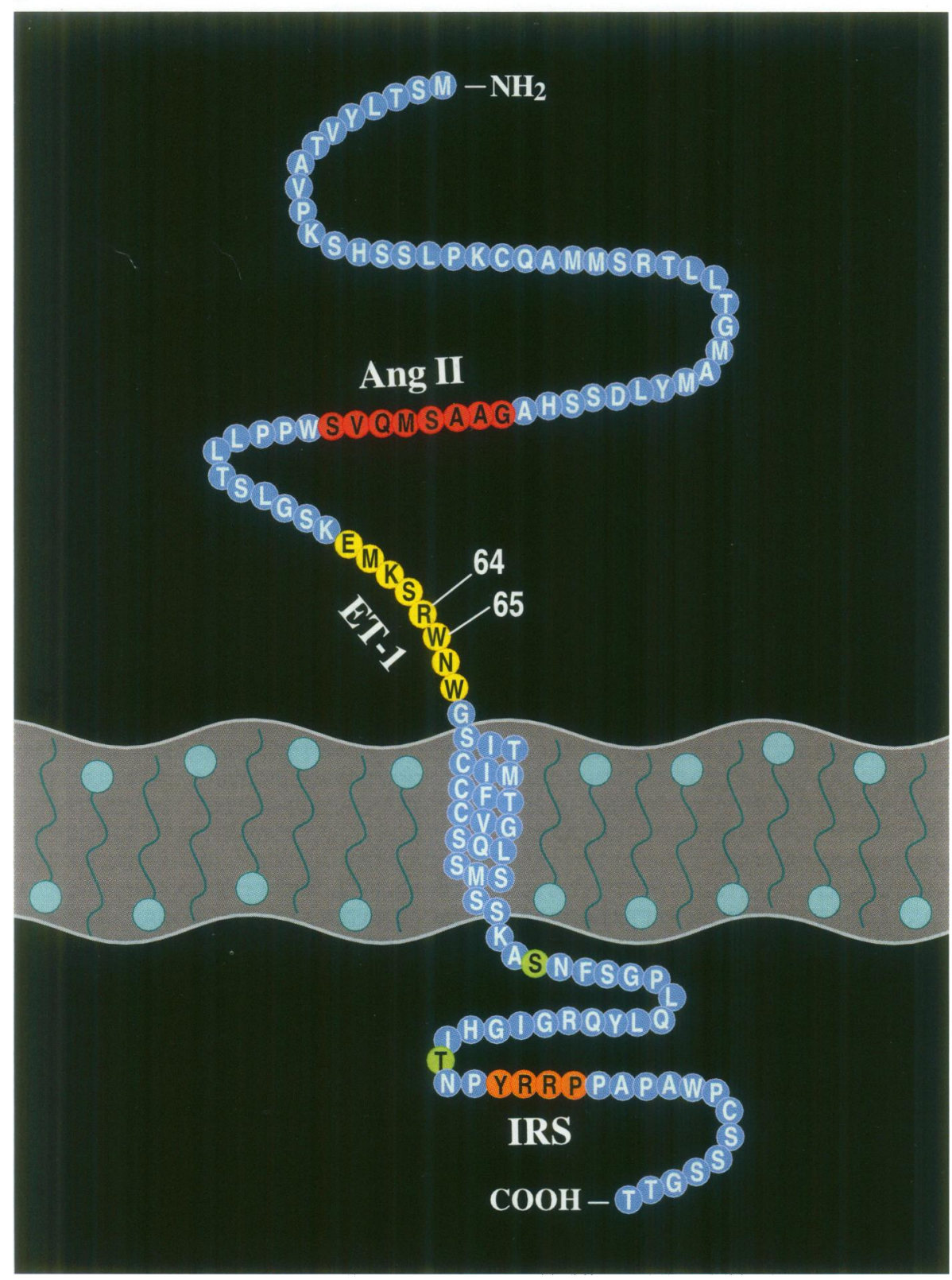

Fig. 1. Sequences and structural features of the ET-1/AngII receptor. (A) The deduced amino acid sequence is presented underneath the nucleotide sequence with the single hydrophobic $(\mathrm{H}-1) \mathrm{pu}-$ tative transmembrane domain underlined. The serine and threonine residues with consensus sequences for cAMP-dependent protein kinase $(18,19)$ located within the intracellular domain are highlighted. The regions depicting 12/19 homology with the AngII cRNA-based oligonucleotide probe [1] and 15/23 homology with the ET-1 cRNA-based oligonucleotide probe [2] are marked; identical nucleotides are dotted. Putative AngII and ET-1 binding domains, and internalization recognition sequences (IRS) are bracketed. Amino acids identical to antisense peptide and conservative amino acid substitutions are highlighted (boldface) within corresponding putative binding domains. *, denotes stop codon. (B) Schematic structure of the ET-1/AngII receptor depicting the extracellular, transmembrane, and cyto- plasmic domains. The following functional domains are highlighted: putative AngII binding domain, AngII, amino acids 41-48 (red); putative ET-1 binding site, ET-1, amino acids 60-67 (yellow); potential cAMP-dependent protein kinase phosphorylation sites $\left(\mathrm{S}_{91}, \mathrm{~T}_{108}\right)$ (green), a potential IRS,

$\left[Y_{111} R R P_{114}\right]$ (orange), showing homology to the human lysosomal acid phosphatase IRS (21); and amino acids $R_{64}$ and $W_{65}$ involved in the site directed mutagenesis. (C) Comparison of the ET-1 and AngII cRNA sequences with the nucleotide and amino acid sequence of the ET-1/AngII receptor identifies $a^{60-67}$ and ${a a_{41-47}}_{4}$ as potential ET- 1 and AngII binding domains, respectively. The stippled areas indicate regions of homology. Nucleotides encompassing the ET-1/AngII receptor ET-1 and AngII binding domains that are present in identical codon position within the corresponding antisense peptide (AP) frames are indicated by asterisks. 


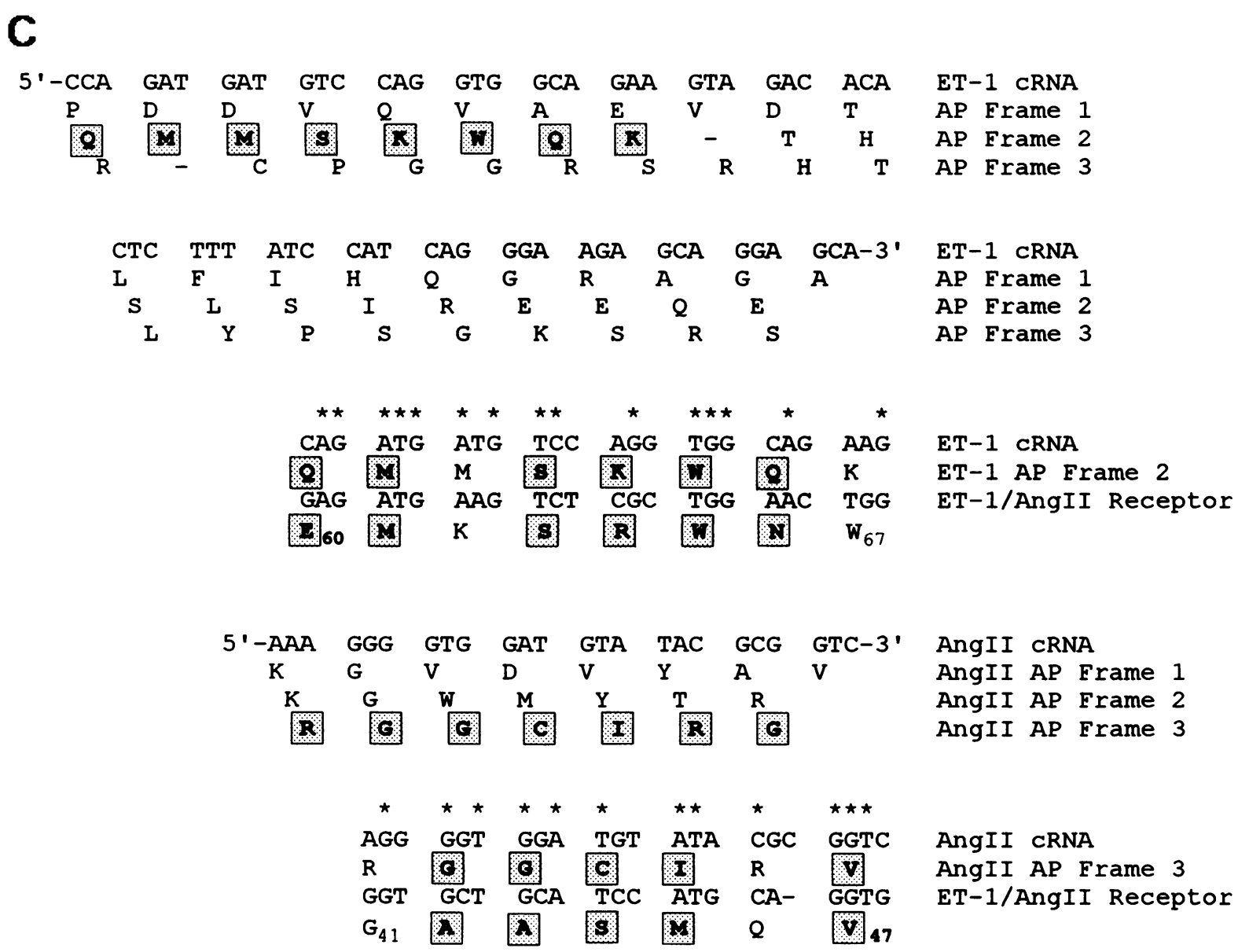

Fig. 1

dependent protein kinase, $S_{91}$ and $T_{108}(18,19)$, can be noted in the predicted cytoplasmic carboxyl-end (Fig. 1). A potential internalization recognition sequence (IRS) is also found in the cytoplasmic carboxyl-end: $\mathrm{Y}_{111} \mathrm{RRP}_{114}$ (Fig. 1), resembling the IRS present in human lysosomal acid phosphatase $(20,21)$. The structural features of the ET-1/AngII polypeptide are consistent with a single-transmembrane dual hormone receptor containing distinct ET-1 and AngII binding domains.

\section{Functional Analysis of the ET-1/AngII Receptor}

Although no other significant ORFs were detected in the cDNA, because of the unusual structure of the ET-1/AngII receptor and the localization of the ET-1/AngII receptor ORF to the $3^{\prime}$ end (Fig. 1A), we tested the possibility that this mRNA could be polycistronic in nature, thus encoding additional polypeptides within the long 5' UT (approximately $2.7 \mathrm{~kb}$ ) necessary for re- ceptor function. For this purpose, two different expression vectors were constructed. The 3274-bp ET-1/AngII receptor cDNA was subcloned directionally ( $5^{\prime}$ to $3^{\prime}$ ) into the NheI site of the pMAMneo expression vector (Clontech) to generate pMAM-ET-1/AngII3274. Similarly, a 756-bp SspI-EcoRI fragment spanning nucleotides 2518-3274 of the full-length cDNA (including the 127 aa ORF, Fig. 1A) was subcloned into the pMAMneo expression vector to generate pMAM-ET-1/AngII756. Both expression vectors were stably transfected into Cos 1 cells and assayed for ${ }^{125} \mathrm{I}$-AngII and ${ }^{125} \mathrm{I}$-ET-1 binding. These binding experiments showed equivalent expression of ET-1/AngII receptors in Cos 1 pMAM-ET1/AngII3274 and CoslpMAM-ET-1/AngII756 transfectants (data not shown). These results demonstrate that the predicted 127 aa open reading frame is necessary and sufficient for receptor function and that the 127 aa polypeptide can interact specifically with ET- 1 and AngII as predicted by the structural analysis (Fig. 1). Con- 

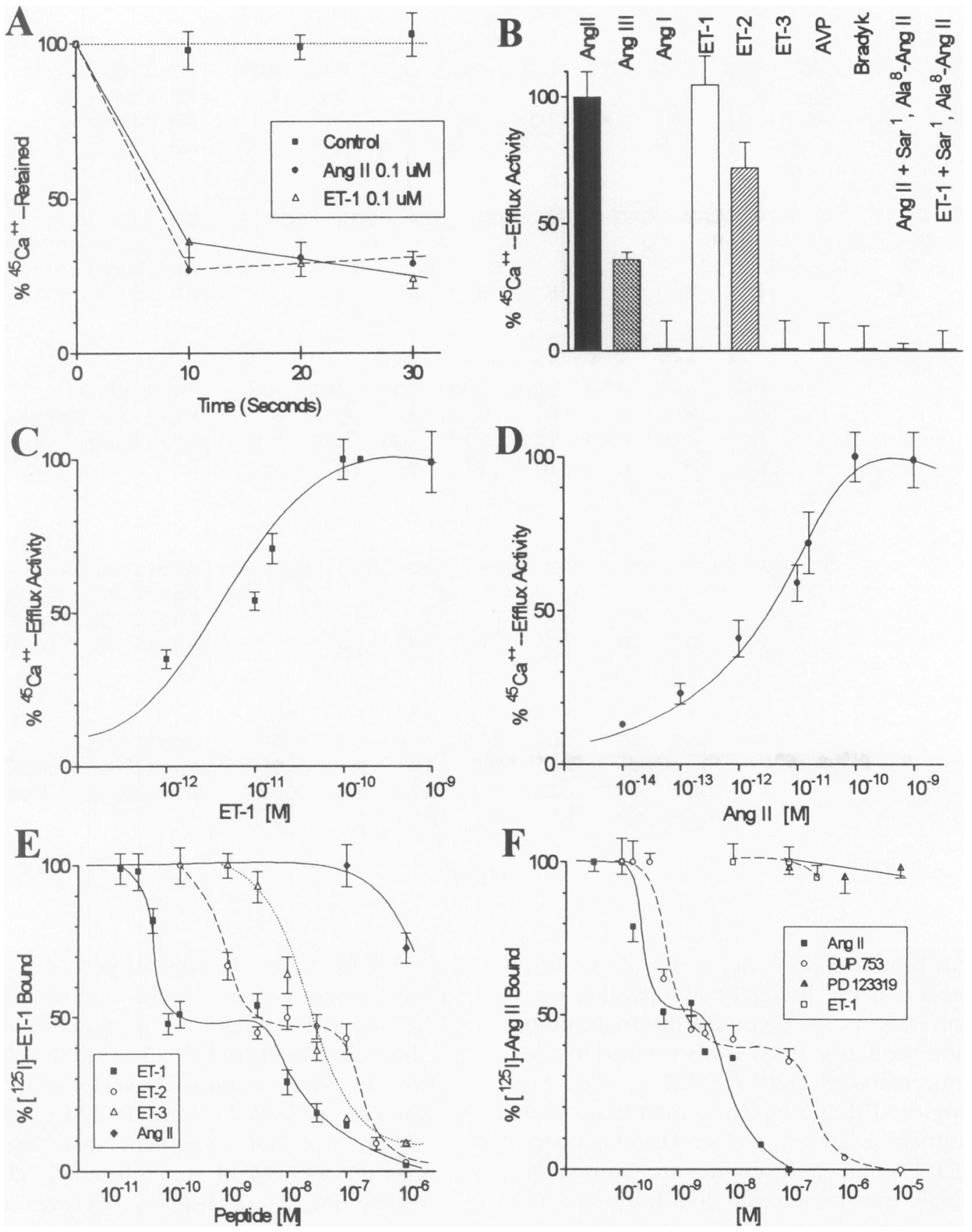

Fig. 2. Functional characterization of the ET-1/ AngII receptor expressed in Cos IpMAM-ET-1/ AngII cells. (A) ET-1 $(0.1 \mu \mathrm{mol} / \mathrm{L})$ and AngII $(0.1$ $\mu \mathrm{mol} / \mathrm{L}$ ) induced ${ }^{45} \mathrm{Ca}^{2+}$ mobilization in intact CoslpMAM-ET-1/AngII cells. ${ }^{45} \mathrm{Ca}^{2+}$ efflux was measured as the $\%{ }^{45} \mathrm{Ca}^{2+}$ retained at 10,20 , and $30 \mathrm{sec}$. (B) Stimulation of ${ }^{45} \mathrm{Ca}^{2+}$ mobilization (measured as $\%{ }^{45} \mathrm{Ca}^{2+}$ efflux activity at $30 \mathrm{sec}$ ) in response to various peptide ligands $(0.1 \mu \mathrm{mol} / \mathrm{L})$ : AngII, AngIII, AngI, ET-1, ET-2, ET-3, AVP, and Bradykinin (Bradyk). Inhibition of ${ }^{45} \mathrm{Ca}^{2+}$ efflux activity by $\mathrm{Sar}^{1}{ }^{1} \mathrm{Ala}^{8}$-AngII was tested with the concurrent incubation of AngII (1 nmol/L) or ET-1 (1 nmol/L)

and $\mathrm{Sar}^{1}{ }^{,} \mathrm{Ala}^{8}$-AngII at $100 \mathrm{nmol} / \mathrm{L}$. (C) Concentration dependence of the ET-1-induced ${ }^{45} \mathrm{Ca}^{2+}$ mobilization (measured as $\%{ }^{45} \mathrm{Ca}^{2+}$-efflux activity at 30 sec). (D) Concentration dependence of the AngIIinduced ${ }^{45} \mathrm{Ca}^{2+}$ mobilization (measured as $\%{ }^{45} \mathrm{Ca}^{2+}$ efflux activity at $30 \mathrm{sec}$ ). (E) Competition for ${ }^{125} \mathrm{I}$ ET-1 specific binding by ET-1 (ם), ET-2 (O), ET-3 $(\triangle)$, and AngII $(\diamond)$. (F) Competition for ${ }^{125}$ I-AngIIspecific binding by AngII ( $\square)$, Losartan (O), PD123319 (A), and ET-1 ( $\square$ ). Each curve is representative of at least three independent experiments performed in quadruplicate. Bars represent the ranges of intraexperimental variation. 
A

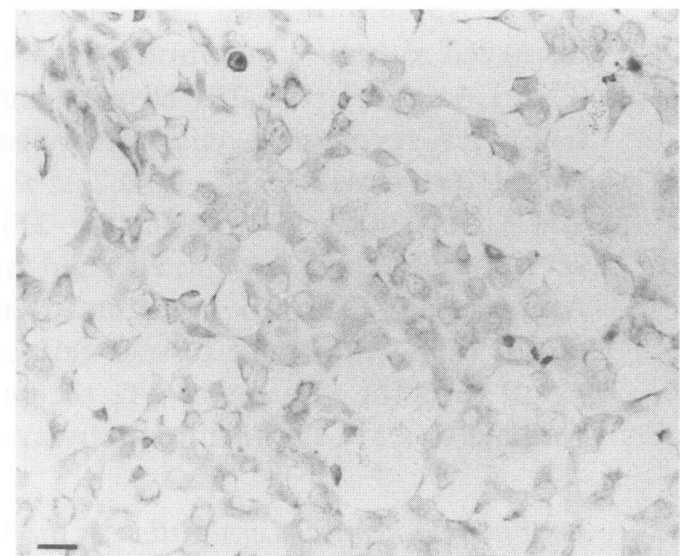

B

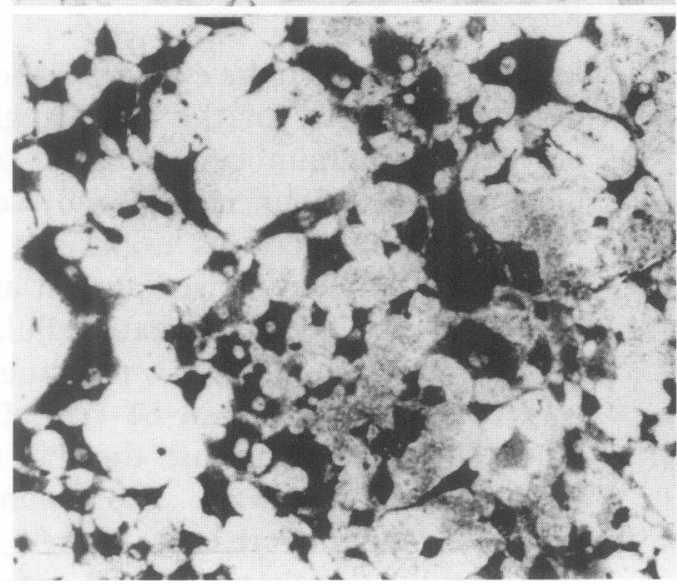

Fig. 3. Immunocytochemical localization of the ET-1/AngII receptor in CosI stable transfectants demonstrating predicted topography and localization to the cell membrane. (A) Control CoslpMAMneo transfectants show minimal background staining. (B) CoslpMAM-ET-1/AngII transfectants show intense immunostaining with the

trol nontransfected and mock-transfected cell did not reveal any binding or activation of second messenger systems upon addition of ET-1 and AngII. All subsequent experiments presented below were conducted with Cos IpMAM-ET-1/ AngII756 transfectants (abbreviated as CoslpMAM-ET-1/AngII).

Because ET- 1 receptors are functionally coupled to a $\mathrm{Ca}^{2+}$ mobilizing transduction system involving phospholipase $\mathrm{C}$ (22), the CoslpMAM-ET-1/AngII transfectants were tested for their ability to support peptide hormone-induced $\mathrm{Ca}^{2+}$ mobilization. Analysis of a panel of peptide hormones revealed that CoslpMAM-ET-1/AngII transfectants responded to ET-1 and AngII (Fig. 2A, B). Measured as the $\%{ }^{45} \mathrm{Ca}^{2+}$ efflux activity indicated by $\%{ }^{45} \mathrm{Ca}^{2+}$ retained (14), $100 \mathrm{nmol} / \mathrm{L} \mathrm{ET-1}$ and $100 \mathrm{nmol} / \mathrm{L}$

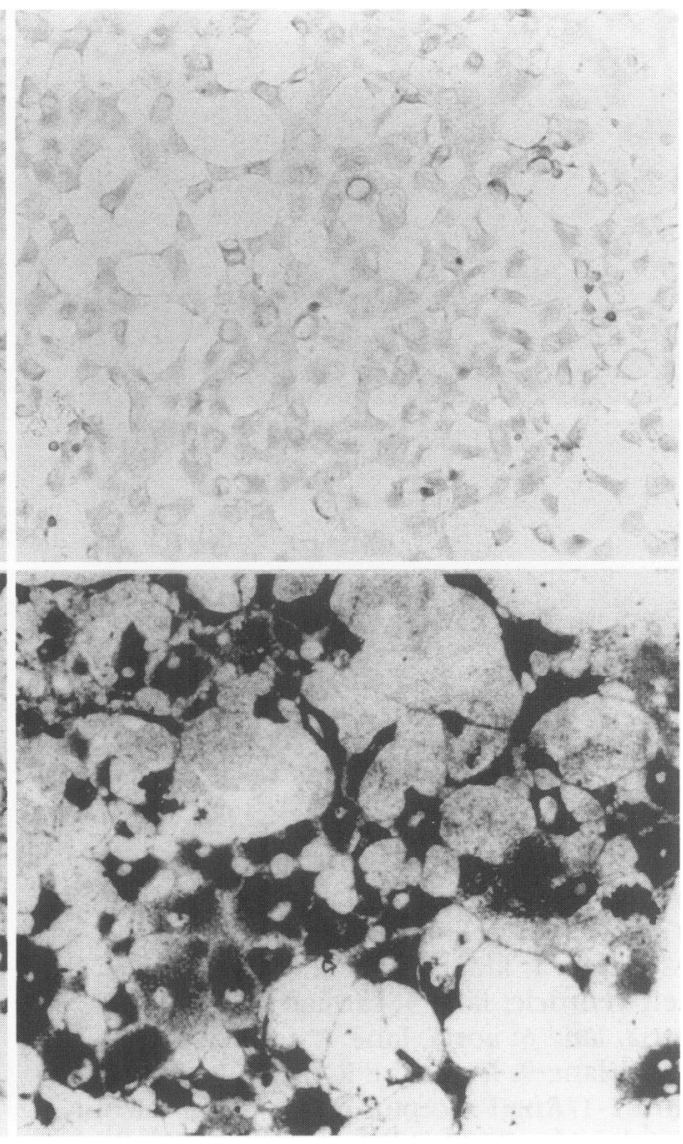

polyclonal antipeptide antibody raised against the predicted extracellular peptide $P_{51}$ LLTSLGSKE $_{60}$ (Fig. 1). Primary antibody was reacted with intact cells followed by immunostaining as described in Materials and Methods. Bar corresponds to $32 \mu \mathrm{M}$ for $\mathrm{A}$ and $\mathrm{B}$.

AngII elicited equivalent amounts of $\mathrm{Ca}^{2+}$ mobilization (Fig. 2B). No responses were elicited by either arginine-vasopressin (AVP) or bradykinin. In comparison to ET-1, ET-2 induced $60 \%$ ${ }^{45} \mathrm{Ca}^{2+}$ efflux activity, whereas ET-3 induced none at all. Similarly, compared with AngII, AngIII induced $25 \%{ }^{45} \mathrm{Ca}^{2+}$ efflux activity, whereas AngI induced none at all (Fig. 2B). The effects of antagonists on the ET-1/AngII receptor were also analyzed. An AngII-specific antagonist (Sar ${ }^{1}, \mathrm{Ala}^{8}$-AngII) efficiently blocked both AngIIand ET-1-induced ${ }^{45} \mathrm{Ca}^{2+}$ efflux (Fig. 2B). This is consistent with the notion that AngII- and ET-1induced responses were mediated by the same receptor.

The specificities of the ET-1 and AngII responses of the dual ET-1/AngII receptor were then assessed in concentration dependence as- 


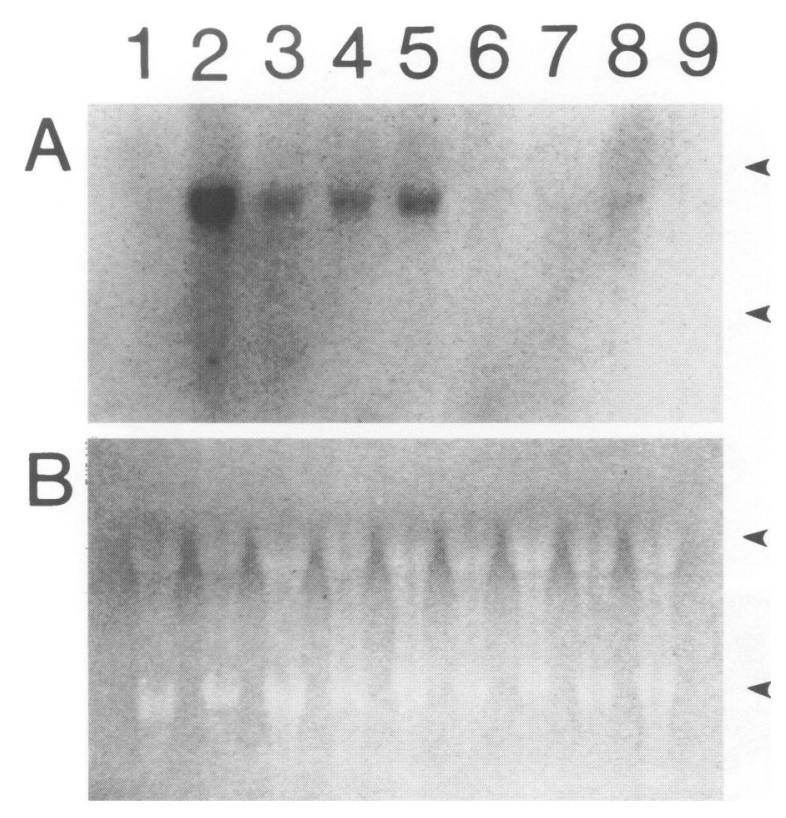

Fig. 4. Tissue distribution of the ET-1/AngII receptor mRNA. RNA blot analysis of different adult rat tissue total cellular RNAs $(5 \mu \mathrm{g})$ is presented (A). Lane 1: kidney, lane 2: brain, lane 3: cardiac left ventricle, lane 4 : cardiac right ventricle, lane 5: atria, lane 6: aorta, lane 7: adrenal gland, lane 8: lung, lane 9: liver. The RNA blot was hybridized to an ET-1/AngII receptor cDNA probe encompassing nucleotides 2520-3274 of the full-length cDNA (Fig. 1) as previously described (41). Nondegradation of RNA samples and relative amounts are directly documented in the ethidium-bromide picture presented in parallel (B). $28 \mathrm{~S}$ and $18 \mathrm{~S}$ ribosomal RNAs are marked by arrowheads.

says measuring ET-1- and AngII-induced ${ }^{45} \mathrm{Ca}^{2+}$ efflux. The response to both ET-l and AngII were similar with equivalent effector concentration for half-maximal response $\left(\mathrm{EC}_{50}\right)$ values of $7 \pm$ $1.4 \mathrm{pmol} / \mathrm{L}$ (Fig. 2C) and $6 \pm 0.9 \mathrm{pmol} / \mathrm{L}$ (Fig. 2D), respectively. Pharmacologic specificity was further ascertained by ligand dissociation studies. As shown in Fig. 2E and F, high $\left(\mathrm{K}_{\mathrm{H}}\right)$ and low $\left(\mathrm{K}_{\mathrm{L}}\right)$ affinity sites were detected for both ET- 1 and AngII with $\mathrm{K}_{\mathrm{H}}$ of $0.05 \pm 0.01 \mathrm{nmol} / \mathrm{L}$ for ET-1 $\left(\mathrm{B}_{\max }=6.0 \pm 1.2 \mathrm{fmol} 10^{-6}\right.$ cells $)$ and $0.27 \pm 0.046 \mathrm{nmol} / \mathrm{L}$ for AngII $\left(\mathrm{B}_{\max }=5.4 \pm 0.8\right.$ fmol $10^{-6}$ cells). The $K_{L}$ for ET-1 was $50 \pm 13.5$ $\mathrm{nmol} / \mathrm{L}\left(\mathrm{B}_{\max }=72 \pm 11.0 \mathrm{fmol} 10^{-6}\right.$ cells $) ; \mathrm{K}_{\mathrm{L}}$ for AngII was $16 \pm 2.4 \mathrm{nmol} / \mathrm{L}\left(\mathrm{B}_{\max }=78 \pm 9.4\right.$ fmol $10^{-6}$ cells). Interestingly, ${ }^{125}$ I-ET-1 was not displaced by AngII, nor was ${ }^{125}$ I-AngII displaced by ET-1 (Fig. 2E, F). Displacement of ET-1 by ET-2 was also detected with high- and low-affinity sites with a $\mathrm{K}_{\mathrm{iH}}$ of $1 \pm 0.13 \mathrm{nmol} / \mathrm{L}$ and $\mathrm{K}_{\mathrm{iL}}$ of
$300 \pm 33 \mathrm{nmol} / \mathrm{L}$ (Fig. 2E). In contrast, ET-3 displayed a single low-affinity site with a $\mathrm{K}_{\mathrm{iL}}$ of $20 \pm 1.6 \mathrm{nmol} / \mathrm{L}$ (Fig. 2E). Displacement of AngII by the $\mathrm{AT}_{1}$ receptor-specific antagonist, Losartan, was detected with a $\mathrm{K}_{\mathrm{iH}}$ of $0.7 \pm 0.14 \mathrm{nmol} / \mathrm{L}$ and $\mathrm{K}_{\mathrm{iL}}$ of $160 \pm 19 \mathrm{nmol} / \mathrm{L}$ (Fig. $2 \mathrm{~F}$ ). The $\mathrm{AT}_{2^{-}}$ specific antagonist, PD123319, did not displace AngII binding up to concentrations of $10^{-5}$ mol/L (Fig. 2F). These results define the ET-1/ AngII receptor as a novel $\mathrm{AT}_{1}$ receptor isoform coupled to $\mathrm{Ca}^{2+}$-mobilizing pathways.

\section{Immunocytochemical Localization of the ET-1/AngII Receptor}

In order to ascertain the cytolocalization and predicted topography of the ET-1/AngII receptor polypeptide, immunocytochemical studies were done on intact (nonpermeabilized) Cos IpMAMET-1/AngII transfectants utilizing a polyclonal antipeptide antibody raised against amino acids 51-60 of the predicted extracellular domain (Fig. 1). As shown in Fig. 3, prominent staining was observed in pMAM-ET-1/AngII transfectants (Fig. 3B). In contrast, the cells transfected with the parental expression vector, CoslpMAMneo, were not stained (Fig. 3A). These results corroborate the predicted topography and cell membrane localization of the ET-1/ AngII receptor.

\section{Tissue Distribution of the ET-1/AngII Receptor} $m R N A$

Analysis of the ET-1/AngII receptor mRNA tissue distribution by Northern blot analysis (Fig. 4) revealed an approximately 4 -kb mRNA prominently expressed in brain and heart tissues. Much lower but detectable levels can be observed in aorta, adrenal gland, and lung tissues. Northern analysis did not detect ET-1/AngII receptor sequences in kidney and liver. However, reverse transcriptase-polymerase chain amplification of $5^{\prime}$ untranslated RNA sequences could detect the ET-1/AngII mRNA in all tissues tested, including kidney and liver (data not shown).

\section{Site-Directed Mutagenesis of the ET-I Binding Domain}

In order to determine whether the putative ET-1 binding domain, as delineated by the homology to antisense peptide sequences (Fig. 1), indeed represents the structural determinants for ET- 1 binding within the ET-1/AngII receptor, we performed mutational studies on the putative ET-1 

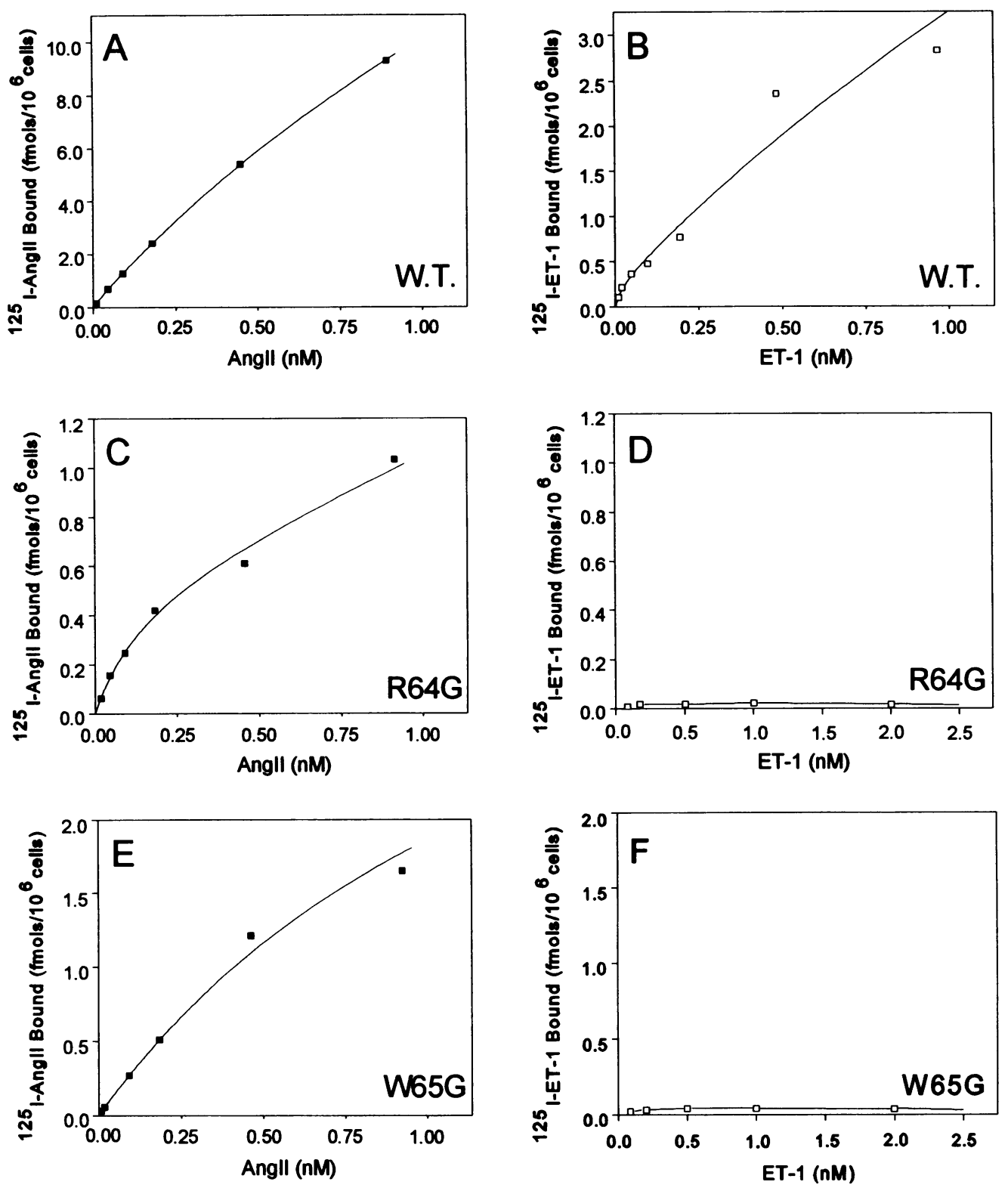

Fig. 5. Assessment of the predicted ET-1/AngII receptor ET-1 binding domain via site-directed mutagenesis. Saturation binding curves with ${ }^{125} \mathrm{I}$ AngII (A, C, E) and ${ }^{125}$ I-ET-1 (B, D, F) in the wildtype (W.T.) (A, B), R64G (C, D), and W65G (E, F)

binding domain while preserving the structure of the putative AngII binding domain. More specifically, our strategy involved modifying the ET-1 binding domain without affecting, or only minimally so, the AngII binding site which would serve as an ideal internal control for potential "unwanted conformation effects" of the mutagenesis directed towards the ET-1 binding domain. We therefore chose to replace $R_{64}$ and

ET-1/AngII receptors expressed in permanent Cos 1 cell transfectants. Mutagenesis and binding studies were performed as described in Materials and Methods. Each curve is representative of at least three independent experiments performed in quadruplicate.

$\mathrm{W}_{65}$, amino acids that contain the largest side chains among the naturally occurring amino acids, for glycine (G), an amino acid that contains the smallest side chain (hydrogen). Such drastic changes in size of the side chain at amino acid positions 64 and 65 were expected to have a prominent effect on the putative conformation of this peptide region with concomitant detrimental effect on ET-1 binding. 
Table 1. Binding parameters of wild-type and mutant ET-1/AngII receptors for AngII and ET-1 expressed in permanent Cosl cell transfectants

\begin{tabular}{lccc}
\hline $\begin{array}{l}\text { ET-1/AngII } \\
\text { Receptor }\end{array}$ & $\begin{array}{c}\mathbf{K}_{\mathbf{H}} \text { ET-1 } \\
(\mathbf{n m o l} / \mathbf{L})\end{array}$ & $\begin{array}{c}\mathbf{K}_{\mathbf{H}} \text { AngII } \\
(\mathbf{n m o l} / \mathbf{L})\end{array}$ & $\begin{array}{c}\mathbf{B}_{\max } \text { for AngII } \\
(\mathbf{f m o l s ~ 1 0}\end{array}$ \\
\hline Wild type & $0.28 \pm 0.07$ & $1.30 \pm 0.30$ & $5.03 \pm 0.99$ \\
R64G & No binding & $1.20 \pm 0.13$ & $0.95 \pm 0.07$ \\
W65G & No binding & $7.36 \pm 1.50$ & $4.69 \pm 0.84$ \\
\hline
\end{tabular}

Scatchard analysis (RADLIG, Version 4 Program, McPherson) of the ${ }^{125}$ I-AngII and ${ }^{125}$ I-ET-1 saturation curves (Fig. 5) was done. $\mathrm{K}_{\mathrm{H}}$, high affinity was stated as $\mathrm{nmol} / \mathrm{L} \pm$ error.

The results of the mutational studies (Fig. 5, Table 1) support the hypothesis that motif $\mathrm{E}_{60} \mathrm{MKSRWNW}_{67}$ is a structural determinant of ET-1 binding in the ET-1/AngII receptor. Substitution of $\mathrm{R}_{64}$ by glycine (R64G; Fig. 5C, D, Table 1) and substitution of W65 by glycine (W65G; Fig. 5E, F, Table 1) completely abrogate ET-1 binding without or minimally affecting AngII binding. It is noted that the R64G substitution did not affect AngII binding (Table 1), whereas W65G substitution decreased the AngII affinity by 5.7 -fold (from wild-type $K_{H}$ of AngII $=1.30 \pm$ $0.30 \mathrm{nmol} / \mathrm{L}$ to $\mathrm{W} 65 \mathrm{G} \mathrm{K}_{\mathrm{H}}$ AngII $=7.36 \pm 1.50$ $\mathrm{nmol} / \mathrm{L}$; Table 1).

\section{Discussion}

The elucidation of the ET-1/AngII receptor as a single transmembrane domain receptor is unique among AngII $(11,23-27)$ and ET $(28-30)$ seventransmembrane domain G-protein-coupled receptors. The dual ET-1/AngII receptor is functionally coupled to a $\mathrm{Ca}^{2+}$-mobilizing transduction system, responding equivalently to both ET-1 and AngII in a highly specific manner. The ET- 1 and AngII EC ${ }_{50}$ values of $7 \mathrm{pmol} / \mathrm{L}$ and $6 \mathrm{pmol} / \mathrm{L}$, respectively, strongly suggest that this receptor is fully functional under normal physiological conditions as their respective $\mathrm{EC}_{50}$ values fall well within the range of normal circulating levels of respective peptide hormones $(31,32)$. Interestingly, Sar ${ }^{1}-\mathrm{Ala}^{8}$-AngII (AngIIspecific antagonist) blocked both the AngII- and ET-1-induced $\mathrm{Ca}^{2+}$ response (Fig. 2B). The molecular mechanism involved in the inhibition of the ET- 1 response by the AngII-specific inhibitor, Sar $^{1}$-Ala ${ }^{8}$-AngII, most likely involves inhibition of ET-1-induced receptor conformational transi- tion necessary to activate the downstream transducing system effected by ET-l since neither AngII (Fig. 2E) nor $\mathrm{Sar}^{1}-\mathrm{Ala}^{8}$-AngII (data not shown) were able to displace ${ }^{125}$ I-ET-1-specific binding.

ET- 1 and AngII can evoke a number of similar responses in different organ systems (33). The existence of the dual ET-1/AngII receptor could partially explain these observations. Recently, it has been reported that both ET-1 and AngII stimulated fibronectin and type IV collagen mRNA expression and mitogenesis in rat mesangial cells (34). Unexpectedly, Losartan (a specific $\mathrm{AT}_{1}$ receptor antagonist) inhibited the ET-1-mediated effects whereas BQ-123 (a specific ET $_{A}$ receptor antagonist) inhibited the AngII-induced fibronectin synthesis and mesangial cell proliferation (34). These data could well be explained by the presence and mediation of these physiologic responses by the ET-1/AngII receptor in rat mesangial cells. The inhibition of ET-1-mediated responses by Losartan (34) resembles the inhibition of ET-1-induced $\mathrm{Ca}^{2+}$ mobilization by Sar ${ }^{1}$ $\mathrm{Ala}^{8}$-AngII noted above (Fig. 2B). We have detected ET-1/AngII receptor mRNA in kidney; however, its existence in mesangial cells remains to be determined.

Our results demonstrate that the motif $\mathrm{E}_{60} \mathrm{MKSRWNW}_{67}$ is necessary for ET-1 binding to the ET-1/AngII receptor. This is in contrast with previous structure-functional studies in which extensive regions within endothelin receptors spanning extracellular, transmembrane, and intracellular portions of the receptor have been invoked to be involved in endothelin binding (35-37). These studies, however, did not exclude the possibility of conformational effects induced by the mutagenic changes because of the lack of "internal control" that could account for 
induced conformational/allosteric effects. It is interesting to note that the antisense peptide delineating the ET-1 binding domain encompasses the carboxyl-terminal 8 amino acids of the ET- 1 peptide, thus indicating that these 8 residues most likely represent the motif within ET-1 that interact with the ET-1/AngII receptor. This is consistent with previous observations implicating the carboxyl-terminal domain of endothelin peptides in their binding to their corresponding receptors $(38-40)$, respectively.

The isolation and characterization of the ET1/AngII receptor elucidates a new paradigm involved in the evolution of hormone-binding domains in different receptors, thus refining a principle of the molecular recognition theory. The data presented above identified the antisense peptides from frames 2 and 3 as the related evolutionary precursor binding domains for ET-1 and AngII, respectively, in the ET-1/AngII receptor (Fig. 1). On the other hand, the antisense peptides in frame 1 for both AngII and AVP were delineated as the corresponding precursor binding domains within the AngII/AVP receptor (11). These findings suggest that antisense peptides originating from the three possible frames can be used as potential precursor binding domains in the evolutionary pathway of different isoreceptors. This intrinsic property of derived antisense peptides probably had a significant impact in the evolution of peptide hormone-cognate receptor systems by effectively increasing the possibility of producing putative cognate receptors. We anticipate that as more molecular genetic, physicochemical, and crystallographic evidence accumulates in the near future, a more complete understanding of the evolution and principles underlying peptide and protein-protein interaction will emerge, thus opening a window into a fundamental organizational principle that governs structure and function in living cells.

\section{Acknowledgments}

We thank Dr. Joan Keiser (Parke-Davis) for providing us with PD123319, Dr. Ronald D. Smith (Dupont Merck) for providing us with Losartan potassium, and A. Tsikoudakis for preparation of the graphs and manuscript.

\section{References}

1. Blalock JE. (1995) Genetic origins of protein shape and interaction rules. Nature Med. 1: 876878.
2. Bost KL, Smith EM, Blalock JE. (1985) Similarity between the corticotropin (ACTH) receptor and a peptide encoded by an RNA that is complementary to ACTH mRNA Proc. Natl. Acad. Sci. U.S.A. 82: 1372-1375.

3. De Souza SJ, Brentani R. (1992) Collagen binding site in collagenase can be determined using the concept of sense-antisense peptide interactions. J. Biol. Chem. 267: 13763-13767.

4. Baranyi L, Campbell W, Ohshima K, Fujimoto S, Boros M, Okada H. (1995) The antisense homology box: A new motif within proteins that encodes biologically active peptides. Nature Med. 1: 894901.

5. Martins VR, Graner E, Garcia-Abreu J, et al. (1997) Complementary hydropathy identifies a cellular prion protein receptor. Nature Med. 3: 1376-1382.

6. Rasmussen UB, Hesch RD. (1987) On antisense peptides: The parathyroid hormone as an experimental example and a critical theoretical view. Biochem. Biophys. Res. Commun. 149: 930-938.

7. de Gasparo M, Whitebread S, Einsle K, Heusser C. (1989) Are the antibodies to a peptide complementary to angiotensin II useful to isolate the angiotensin II receptor? Biochem. J. 261: 310-311.

8. Golstein A, Brutlag DL. (1989) Is there a relationship between DNA sequences encoding peptide ligands and their receptors? Proc. Natl. Acad. Sci. U.S.A. 86: 42-45.

9. Kelly JM, Trinder D, Phillips PA, et al. (1990) Vasopressin antisense peptide interactions with the V1 receptor. Peptides 11: 857-862.

10. Jurzak M, Pavo I, Fahrenholz F. (1993) Lack of interaction of vasopressin with its antisense peptides: A functional and immunological study. $J$. Recept. Res. 13: 881-902.

11. Ruiz-Opazo N, Akimoto K, Herrera VLM. (1995) Identification of a novel dual angiotensin II/vasopressin receptor on the basis of molecular recognition theory. Nature Med. 1: 1074-1081.

12. Yanagisawa $M$, Kurihara $H$, Kimura $S$, et al. (1988) A novel potent vasoconstrictor peptide produced by vascular endothelial cells. Nature 332: 411-415.

13. Ohkubo H, Kageyama R, Ujihara $M$, Hirose $T$, Inayama I, Nakanishi S. (1983) Cloning and sequence analysis of cDNA for rat angiotensinogen. Proc. Natl. Acad. Sci. U.S.A. 80: 2196-2200.

14. Brown RD, Berger KD, Taylor P. (1984) $\alpha_{1}$-adrenergic receptor activation mobilizes cellular $\mathrm{Ca}^{++}$ in a muscle cell line. J. Biol. Chem. 259: 75547562.

15. Kyte J, Doolittle RF. (1982) A simple method for displaying the hydropathic character of a protein. J. Mol. Biol. 157: 105-132.

16. Engelman DM, Steitz TA, Goldman A. (1986) Identifying nonpolar transbilayer helices in amino acid sequences of membrane proteins. Annu. Rev. Biophys. Biophys. Chem. 15: 321-353. 
17. Adams GA, Rose JK. (1985) Structural requirements of a membrane-spanning domain for protein anchoring and cell surface transport. Cell 41: 1007-1015.

18. Kemp BE, Pearson RB. (1990) Protein kinase recognition sequence motifs. Trends Biochem. Sci. 15: 342-346.

19. Lytle C, Forbush B 3d. (1992) The Na,K,Cl-cotransport protein of shark rectal gland. II. Regulation by direct phosphorylation. J. Biol. Chem. 267: 25438-25443.

20. Trowbridge IS. (1991) Endocytosis and signals for internalization. Curr. Opin. Cell Biol. 3: 634-641.

21. Peters C, Braun M, Weber B, et al. (1990) Targeting of a lysosomal membrane protein: A tyrosinecontaining endocytosis signal in the cytoplasmic tail of lysosomal acid phosphatase is necessary and sufficient for targeting to lysosomes. $E M B O \mathrm{~J}$. 9: 3497-3506.

22. Simonson MS, Dunn MJ. (1990) Cellular signaling by peptides of the endothelin gene family. FASEB J. 4: 2989-3000.

23. Murphy TJ, Alexander RW, Griendling KK, Runge MS, Bernstein KE. (1991) Isolation of a cDNA encoding the vascular type-1 angiotensin II receptor. Nature 35: 233-236.

24. Sasaki K, Yamano Y, Bardhan S, et al. (1991) Cloning and expression of a complementary DNA encoding a bovine adrenal angiotensin II type-1 receptor. Nature 351: 230-233.

25. Sanberg K, Ji H, Clark AJL, Shapira H, Catt KJ. (1992) Cloning and expression of a novel angiotensin II receptor subtype. J. Biol. Chem. 267: 9455-9458.

26. Kambayashi Y, Bardhan S, Takahashi K, et al. (1993) Molecular cloning of a novel angiotensin II receptor isoform involved in phosphotyrosine phosphatase inhibition. J. Biol. Chem. 268: 2454324546.

27. Mukoyama $M$, Nakajima $M$, Horiuchi $M$, Sasamura H, Pratt RE, Dzau VJ. (1993) Expression cloning of the type 2 angiotensin II receptor reveals a unique class of seven-transmembrane receptor. J. Biol. Chem. 268: 5439-5442.

28. Sakurai T, Yanagisawa M, Takuwa Y, et al. (1990) Cloning of a cDNA encoding a nonisopeptide-selective subtype of the endothelin receptor. Nature 348: $732-735$.

29. Lin HY, Kaji EH, Winkel GK, Ives HE, Lodish HF. (1991) Cloning and functional expression of a vascular smooth muscle endothelin-1 receptor. Proc. Natl. Acad. Sci. U.S.A. 88: 3185-3189.
30. Karne S, Jayawickreme CK, Lerner MR. (1993) Cloning and characterization of an endothelin-3 specific receptor $\left(\mathrm{Et}_{\mathrm{c}}\right.$ receptor) from Xenopus laevis dermal membranes. J. Biol. Chem. 268: 1912619133.

31. Powell-Jackson JD, MacGregor J. (1976) Radioimmunoassay of angiotensin II in the rat. J. Endocrinol. 68: 175-176.

32. Battistini B, D'Orleans-Juste P, Sirois P. (1993) Endothelins: circulating plasma levels and presence in other biologic fluids. Lab. Invest. 68: 600628.

33. Egido J. (1996) Vasoactive hormones and renal sclerosis: Nephrology forum. Kidney Int. 49: 578597.

34. Gomez-Garre D, Ruiz-Ortega M, Ortego $M$, et al. (1996) Effects and interactions of endothelin-1 and angiotensin II on matrix protein expression and synthesis and mesangial cell growth. Hypertension 27: 885-892.

35. Hashido K, Gamou T, Adachi M, et al. (1992) Truncation or N-terminal extracellular of C-terminal intracellular domains of human $\mathrm{ET}_{\mathrm{A}}$ receptor abrogated the binding activity of ET-1. Biochem. Biophys. Res. Commun. 187: 1241-1248.

36. Adachi M, Yang Y, Trezciak A, Furuichi Y, Miyamoto C. (1992) Identification of a domain of $\mathrm{ET}_{\mathrm{A}}$ receptor required for ligand binding. FEBS Lett. 311: 179-183.

37. Hashido $K$, Adachi M, Gamou T, Watanabe $T$, Furuichi Y, Miyamoto C. (1993) Identification of specific intracellular domains of the human $\mathrm{ET}_{\mathrm{A}}$ receptor required for ligand binding and signal transduction. Cell. Mol. Biol. Res. 39: 3-12.

38. Kimura S, Kasuya Y, Sawamura T, et al. (1988) Structure-activity relationships of endothelin: Importance of C-terminal moiety. Biochem. Biophys. Res. Commun. 156: 1182-1186.

39. Maggi CA, Giuliani S, Patacchini R, et al. (1989) The C-terminal hexapeptide, endothelin-(16-21), discriminates between different endothelin receptors. Eur. J. Pharmacol. 166: 121-122.

40. Takai M, Umemura I, Yamasaki K, et al. (1992) A potent and specific agonist, Suc-[Glu9, Ala 1 1,15]endothelin-1(8-21), IRL 1620, for the ETB receptor. Biochem. Biophys. Res. Commun. 184: 953-959.

41. Herrera VLM, Chobanian AV, Ruiz-Opazo N. (1988) Isoform-specific modulation of $\mathrm{Na}^{+}, \mathrm{K}^{+}$. ATPase $\alpha$-subunit gene expression in hypertension. Science 241: 221-223. 